\title{
An Adaptive Nonmonotone Line Search Technique for Solving Systems of Nonlinear Equations
}

\author{
Masoud Hatamian $\mathbb{D}^{1},{ }^{1}$ Mahmoud Paripour $\mathbb{D}^{2},{ }^{2}$ Farajollah Mohammadi Yaghoobi $\mathbb{D}{ }^{1}$ \\ and Nasrin Karamikabir ${ }^{1}{ }^{1}$ \\ ${ }^{1}$ Department of Mathematics, Hamedan Branch, Islamic Azad University, Hamedan, Iran \\ ${ }^{2}$ Department of Computer Engineering and Information Technology, Hamedan University of Technology, Hamedan, Iran
}

Correspondence should be addressed to Mahmoud Paripour; m_paripour@yahoo.com

Received 28 May 2021; Revised 12 August 2021; Accepted 14 August 2021; Published 27 August 2021

Academic Editor: Mehdi Ghatee

Copyright (C) 2021 Masoud Hatamian et al. This is an open access article distributed under the Creative Commons Attribution License, which permits unrestricted use, distribution, and reproduction in any medium, provided the original work is properly cited.

In this article, a new nonmonotone line search technique is proposed for solving a system of nonlinear equations. We attempt to answer this question how to control the degree of the nonmonotonicity of line search rules in order to reach a more efficient algorithm? Therefore, we present a novel algorithm that can avoid the increase of unsuccessful iterations. For this purpose, we show the robust behavior of the proposed algorithm by solving a few numerical examples. Under some suitable assumptions, the global convergence of our strategy is proved.

\section{Introduction}

Different ranges of sciences such as economics, chemistry, robotics, and engineering can be formulated as systems of nonlinear equations. On the other hand, scientific advances in these areas are more dependent on the resolution of these systems.

There are two kinds of system equations that are called linear and nonlinear systems and there are two primary methods for solving them called numerical solutions and optimization methods. Optimization-based methods are the most widely used ones for solving the nonlinear type.

So, in this article, we discuss how to find solutions to a system of nonlinear equations as follows:

$$
F(x)=0 \text {, }
$$

where $F: \mathbb{R}^{n} \longrightarrow \mathbb{R}^{m}$ is continuously differentiable map and $m \leq n$. Let the form of (1) be

$$
\left\{\begin{array}{l}
F_{1}\left(x_{1}, x_{2}, \ldots, x_{n}\right)=0, \\
F_{2}\left(x_{1}, x_{2}, \ldots, x_{n}\right)=0, \\
\vdots \\
F_{m}\left(x_{1}, x_{2}, \ldots, x_{n}\right)=0 .
\end{array}\right.
$$

In recent years, several optimization-based methods have been developed to solve the nonlinear system of equation (1) such as Newton class and gradient class. The Newton class, for example, Newton and quasi-Newton methods, is more suitable for the small-scale systems of equations and gradient class; for example, the spectral gradient, and conjugate gradient methods, can suitably cope with large-scale systems. There are different approaches to converting the problem (1) to an optimization problem. For example, in the method proposed in [1], the authors transformed the system into an unconstrained optimization problem and then solved the new unconstrained problem instead by applying an optimization method that is formulated as follows: 


$$
\begin{aligned}
& f=F^{2}(x)=F_{1} F_{1}^{T}+F_{2} F_{2}^{T}+\cdots+F_{m} F_{m}^{T} \geq 0, \\
& f=|F(x)|=\left|F_{1}\right|+\left|F_{1}\right|+\cdots+\left|F_{m}\right| \geq 0,
\end{aligned}
$$

and another forms exist $[2,3]$. In this article, we consider the following form:

$$
f(x)=\left(\frac{1}{2}\right)\|F(x)\|^{2},
$$

where $\|$.$\| stands for the Euclidean norm. So we replace the$ problem (1) by

$$
\min _{x \in \mathbb{R}^{n}} f(x),
$$

and set $g(x)=\nabla f(x)$. Indubitably, optimal solutions of problem (5) with the zero value of the objective function correspond to global solutions of system (1). Most of the optimization-based methods can be divided into two classes, namely, line search methods and trust-region methods $[3,4]$. A line search method, at current iteration $x_{k}$ if $g_{k}=\nabla f\left(x_{k}\right) \neq 0$, defines a search direction $d_{k}$ in some way and finds a step-length by carrying some line search along $d_{k}$. The most popular line search rules are the Armijo rule, the Goldstein rule, and the Wolfe rule [5-7]. For a given $x_{k}$, the line search generates the next point by

$$
x_{k+1}=x_{k}+\alpha_{k} d_{k}
$$

where $\alpha_{k}>0$ is a step size obtained by the line search and $d_{k}$ is a descent direction. The traditional line searches require the function value descent monotonically at every iteration, namely,

$$
f\left(x_{k+1}\right) \leq f\left(x_{k}\right) .
$$

The monotone rule may extremely decrease the rate of convergence when the iteration is ensnared near a narrow curved valley, which can cause very short steps or zigzagging. Grippo et al. [8] proposed the earliest nonmonotone line search. By combining forcing function with the nonmonotone line search techniques, Sun et al. [9] proposed a general nonmonotone line search rule, called nonmonotone F-rule, for unconstrained optimization. For solving systems of equations, Ataee Tarzanagh et al. in [4] and Reza Peyghami and Ataee Tarzanagh in [10] developed a new nonmonotone term according to

$$
T_{k}=\left(1+\eta_{k}\right) R_{k}=\left(1+\eta_{k}\right)\left(\varepsilon_{k} f_{l(k)}+\left(1-\varepsilon_{k}\right) f_{k}\right),
$$

where $\varepsilon_{k} \in\left[\varepsilon_{\min }, \varepsilon_{\max }\right] \subset[0,1]$ and $f_{k}=f\left(x_{k}\right)$. Moreover, $f_{l(k)}$ is Grippo's nonmonotone term which is defined by

$$
f_{l(k)}=\max _{0 \leq j \leq l(k)} f_{k-j},
$$

where $l(0)=0$, and for $k \geq 1,0 \leq l(k) \leq \min \{k, M\}$, where $M$ is a given positive integer, and $\eta_{k}$ is a vanishing positive sequence satisfying the following condition:

$$
\sum_{k=1}^{\infty} \eta_{k}=\eta<\infty
$$

Note that, for

$$
R_{k}=\varepsilon_{k} f_{l(k)}+\left(1-\varepsilon_{k}\right) f_{k},
$$

they proved the weak convergence property

$$
\liminf _{k \longrightarrow \infty}\left\|g_{k}\right\|=0
$$

The main contribution of the proposed algorithm in this article is to use function derived value to improve the sequencing process. We introduce an adaptive nonmonotone technique which has a strong convergence property,

$$
\lim _{k \longrightarrow \infty}\left|g_{k}\right|=0,
$$

and can control the convex combination of function values better than previous methods. Numerical experiments will show the efficiency of the proposed line search technique. Numerical experiments indicate the efficiency and robustness of the new proposed technique.

The remainder of this article is organized as follows: In Section 2, we present our proposed adaptive nonmonotone rule and in Section 3, we present the convergence property it. The numerical results and conclusions are given in Sections 4 and 5, respectively.

\section{Algorithmic Structure}

The following assumption is imposed throughout the article.

Assumption 1. $f(x)$ is bounded below on the level set $\mathscr{L}=$ $\left\{x \in \mathbb{R}^{n} \mid f(x) \leq f\left(x_{0}\right)\right\}$ and $\nabla f(x)$ is continuous uniformly on an open set that contains the level set $\mathscr{L}$.

In order to attain global convergence, we need the search direction satisfying the following assumption.

Assumption 2.

$$
\left|\frac{-g_{k}^{T} d_{k}}{\left\|d_{k}\right\|}\right| \geq \sigma\left(\left\|g_{k}\right\|\right), \quad k=1,2, \ldots,
$$

where $\sigma(\cdot)$ is a forcing function that we bring it later.

Assumption 3. The matrix $J(x)$ is uniformly nonsingular on $\mathscr{L}$, i.e., there exists a constant $M_{0}>0$ such that

$$
M_{0}\|F(X)\| \leq\left\|J(x)^{T} F(x)\right\|=\|g(x)\|, \quad \forall x \in \mathscr{L} .
$$
[9].

In the following, we give forcing function definition as in

Definition 1. The function $\sigma:[0,+\infty) \longrightarrow[0,+\infty)$ is a forcing function ( $F$-function) if for any sequence $\left\{t_{i}\right\} \subset[0,+\infty)$

$$
\lim _{i \longrightarrow \infty} \sigma\left(t_{i}\right)=0 \text { implies } \lim _{i \longrightarrow \infty} t_{i}=0 .
$$
follows:

We define the new nonmonotone line search method as

$$
f\left(x_{k}+\alpha_{k} d_{k}\right) \leq \mathscr{H}_{k}-\sigma\left(t_{k}\right)
$$

set $x_{k+1}=x_{k}+\alpha_{k} d_{k}$, that 


$$
\begin{aligned}
& \mathscr{H}_{k}=\left(1+\mu_{k}\right) C_{k}=\left(1+\mu_{k}\right)\left\{\begin{array}{l}
\left\|g_{k}\right\|^{k} f_{\ell_{k}}+\left(1-\left\|g_{k}\right\|^{k}\right) f_{k}, \quad\left\|g_{k}\right\| \leq 1, \\
\left(1-\left\|g_{k}\right\|^{-k}\right) f_{\ell_{k}}+\left\|g_{k}\right\|^{-k} f_{k}, \quad\left\|g_{k}\right\|>1,
\end{array}\right. \\
& f_{\ell_{k}}=\sum_{r=0}^{\ell_{k}-1} \lambda_{k r} f\left(x_{k-r}\right),
\end{aligned}
$$

where $\quad \ell_{k}=\min \left\{k, M_{k}\right\}, \lambda_{k r} \geq \lambda, r=1,2, \ldots, \ell_{k}-1$, $\lambda \in(0,1]$ and $\sum_{r=0}^{\ell_{k}^{k-1}} \lambda_{k r}=1$, that

$$
M_{k}= \begin{cases}{\left[\left\|g_{k}\right\|+c\right],} & \left\|g_{k}\right\|<10 \\ 10, & \left\|g_{k}\right\| \geq 10\end{cases}
$$

where [.] denotes the floor function and $0<c<1$, and $\left\{\mu_{k}\right\}$ is a vanishing positive sequence satisfying the following condition:

$$
\sum_{k=1}^{\infty} \mu_{k}=\mu<\infty
$$

and $\sigma\left(t_{k}\right)$ is a forcing function and $t_{k}=g_{k}^{T} d_{k} /\left\|d_{k}\right\|$. So

$$
f_{\alpha} \leq \mathscr{H}_{k}+\alpha \rho g_{k}^{T} d_{k},
$$

where $0<\rho<1$. By using Definition 2.3. and Proposition 2.4. in [9], the nonmonotone line search rules (21) easily satisfy the new nonmonotone rule (17). $M$ is updated by (19) in each iteration. We present now a high-level description of our proposed algorithm called adaptive nonmonotone line search technique (ANLS).

With variable $J$ in Algorithm 1, we try to avoid the extra iteration.

\section{Global Convergence}

In this section, we render the global convergence of our algorithm. In our proofs, we employ the method used in [11].

Lemma 1. Let sequence $\left\{x_{k}\right\}_{k \geq 0}$ be a sequence generated by Algorithm 1; then the following inequality holds:

$$
f\left(x_{k}\right) \leq\left(1+\mu_{0}\right)^{k} f\left(x_{0}\right)-\lambda \sum_{r=0}^{k-1} \sigma\left(t_{r}\right) \text {. }
$$

Proof. Let us begin our proof by induction.

If $k=1$, from (17), we have from $\lambda \leq 1$ that

$$
f\left(x_{1}\right) \leq\left(1+\mu_{0}\right) f\left(x_{0}\right)-\lambda \sigma\left(t_{0}\right) .
$$

Suppose that (22) holds for all $1,2, \ldots, k$. To continue the proof, we consider two cases:

Case 1: $\left\|g_{k}\right\| \leq 1$

By hypothesis of induction and $-\lambda \sum_{r=0}^{k-2} \sigma\left(t_{r}\right)-$ $\sigma\left(t_{k-1}\right) \leq-\lambda \sum_{r=0}^{k-1} \sigma\left(t_{r}\right)$ for $k \geq 2$ :

$$
\begin{aligned}
f\left(x_{k+1}\right)= & f\left(x_{k}+\alpha_{k} d_{k}\right) \\
\leq & \left(1+\mu_{k}\right)\left[\left\|g_{k}\right\|^{k} \sum_{r=0}^{\ell_{k}-1} \lambda_{k r} f\left(x_{k-r}\right)+\left(1-\left\|g_{k}\right\|^{k}\right) f_{k}\right]-\sigma\left(t_{k}\right) \\
\leq & \left(1+\mu_{k}\right)\left[\left\|g_{k}\right\|^{k} \sum_{p=0}^{q} \lambda_{k p} f\left(x_{k-p}\right)+\left(1-\left\|g_{k}\right\|^{k}\right)\left[\left(1+\mu_{0}\right)^{k} f\left(x_{0}\right)-\lambda \sum_{r=0}^{k-1} \sigma\left(t_{r}\right)\right]\right]-\sigma\left(t_{k}\right) \\
\leq & \left(1+\mu_{k}\right)\left[\left\|g_{k}\right\|^{k} \sum_{p=0}^{q} \lambda_{k p}\left(\left(1+\mu_{0}\right)^{k-p} f\left\{x_{0}\right)-\lambda \sum_{r=0}^{k-p-2} \sigma\left(t_{r}\right)-\sigma\left(t_{k-p-1}\right)\right)+\left(1-\left\|g_{k}\right\|^{k}\right)\right. \\
& \left.\cdot\left[\left(1+\mu_{0}\right)^{k} f\left(x_{0}\right)-\lambda \sum_{r=0}^{k-1} \sigma\left(t_{r}\right)\right]\right]-\sigma\left(t_{k}\right) .
\end{aligned}
$$

By using $(0,1,2, \ldots, q) \times(0,1,2, \ldots, k-q-2) \subset$ $\{(p, r) ; 0 \leq p \leq q, 0 \leq r \leq k-q-2\} \quad$ and $\quad \sum_{p=0}^{q} \lambda_{k p}=1$, $\lambda_{k p} \geq \lambda$, we have 
Input: Given $x_{0} \in \mathbb{R}^{n}$, constant $\varepsilon>0, \sigma>0, \ell_{0}=1, \rho \in(0,1), \beta \in(0,1), J \in \mathbb{N}, N \in \mathbb{N}$; Output: $x^{*} ; f^{*}$;

begin

$k:=0$;

$x_{k+1}=x^{+} ; f_{k}=f\left(x_{k}\right) ; g_{k}=g\left(x_{k}\right) ; H_{k}=H\left(x_{k}\right)$ (Hessian matrix of $\left.f_{k}\right) ;$ while $\left\|f_{k}\right\| \geq \varepsilon$ and $k \leq N$ do

if $H_{k}$ is singular then

$d_{k}=-g_{k}$

else

$d_{k}=-H_{k} \backslash g_{k}(\backslash$ is matrix division);

if $\left|g_{k}^{T} d_{k}\right|<\sigma\left\|d_{k}\right\|\left\|g_{k}\right\|$ then

$d_{k}=-g_{k}$

else

\section{if $g_{k}^{T} d_{k}>0$ then}

$d_{k}=-d_{k}$ or $d_{k}=-0.5\left(d_{k}+g_{k}\right)$

end

end

$\alpha=1 ; j=0$; compute $f_{\alpha}=f\left(x_{k}+\alpha d_{k}\right), \delta=\rho g_{k}^{T} d_{k}$ and $\mathscr{H}_{k} b y(18) ;$

while 1 do

$j=j+1$

if $f_{\alpha} \leq \mathscr{H}_{k}+\alpha \delta$ or $j==J$, break; en d; then

$\alpha:=\beta \alpha ; x^{+}=x_{k}+\alpha d_{k} ;$ compute $f_{\alpha}\left(x^{+}\right)$;

$$
\text { end }
$$

end

$x_{k+1}=x^{+} ; f_{k+1}:=f_{\alpha}\left(x_{k+1}\right) ; \ell_{k}:=\min \left[k+1, M_{k}\right] ; k:=k+1 ;$

end

end

$x^{*}:=x_{k} ; f^{*}:=f\left(x^{*}\right)$

Algorithm 1: ANLS (adaptive nonmonotone line search).

$$
\begin{aligned}
& f\left(x_{k+1}\right) \leq\left(1+\mu_{k}\right)\left[\left\|g_{k}\right\|^{k}\left[\left(1+\mu_{0}\right)^{k-p} f\left(x_{0}\right)-\lambda \sum_{r=0}^{k-q-2}\left(\sum_{p=0}^{q} \lambda_{k p}\right) \sigma\left(t_{r}\right)-\sum_{p=0}^{q} \lambda_{k p} \sigma\left(t_{k-p-1}\right)\right]\right. \\
& \left.+\left(1-\left\|g_{k}\right\|^{k}\right)\left[\left(1+\mu_{0}\right)^{k} f\left(x_{0}\right)-\lambda \sum_{r=0}^{k-1} \sigma\left(t_{r}\right)\right]\right]-\sigma\left(t_{k}\right) \\
& \leq\left(1+\mu_{k}\right)\left[\left\|g_{k}\right\|^{k}\left[\left(1+\mu_{0}\right)^{k-p} f\left(x_{0}\right)-\lambda \sum_{r=0}^{k-q-2} \sigma\left(t_{r}\right)-\lambda \sum_{r=k-q-1}^{k-1} \sigma\left(t_{r}\right)\right]\right. \\
& \left.+\left(1-\left\|g_{k}\right\|^{k}\right)\left[\left(1+\mu_{0}\right)^{k} f\left(x_{0}\right)-\lambda \sum_{r=0}^{k-1} \sigma\left(t_{r}\right)\right]\right]-\sigma\left(t_{k}\right) \\
& =\left(1+\mu_{k}\right)\left[\left\|g_{k}\right\|^{k}\left[\left(1+\mu_{0}\right)^{k-p} f\left(x_{0}\right)-\lambda \sum_{r=0}^{k-1} \sigma\left(t_{r}\right)\right]+\left(1-\left\|g_{k}\right\|^{k}\right)\right. \\
& \left.\cdot\left[\left(1+\mu_{0}\right)^{k} f\left(x_{0}\right)-\lambda \sum_{r=0}^{k-1} \sigma\left(t_{r}\right)\right]\right]-\sigma\left(t_{k}\right) \\
& \leq\left(1+\mu_{k}\right)\left[\left\|g_{k}\right\|^{k}\left[\left(1+\mu_{0}\right)^{k} f\left(x_{0}\right)-\lambda \sum_{r=0}^{k-1} \sigma\left(t_{r}\right)\right]+\left(1-\left\|g_{k}\right\|^{k}\right)\right. \\
& \left.\cdot\left[\left(1+\mu_{0}\right)^{k} f\left(x_{0}\right)-\lambda \sum_{r=0}^{k-1} \sigma\left(t_{r}\right)\right]\right]-\sigma\left(t_{k}\right)
\end{aligned}
$$




$$
\begin{aligned}
& =\left(1+\mu_{k}\right)\left[\left(1+\mu_{0}\right)^{k} f\left(x_{0}\right)-\lambda \sum_{r=0}^{k-1} \sigma\left(t_{r}\right)\right]-\sigma\left(t_{k}\right) \\
& \leq\left(1+\mu_{k}\right)\left[\left(1+\mu_{0}\right)^{k} f\left(x_{0}\right)\right]-\lambda \sum_{r=0}^{k} \sigma\left(t_{r}\right) \\
& \leq\left(1+\mu_{0}\right)^{k+1} f\left(x_{0}\right)-\lambda \sum_{r=0}^{k} \sigma\left(t_{r}\right) .
\end{aligned}
$$

\section{Case 2: $\left\|g_{k}\right\|>1$}

Inasmuch as $\left\|g_{k}\right\|^{-1}<1$, the proof is the same as in the first case.

Now by using Lemma 1, we establish a strong global convergence theorem for our new nonmonotonte algorithm.

Theorem 1. Let assumptions $1-3$ are satisfied and $\left\{x_{k}\right\}$ be a the sequence generated by Algorithm 1. Then $\left\{x_{k}\right\} \subseteq \mathscr{L}$ and

$$
\lim _{k \rightarrow \infty}\left\|F_{k}\right\|=0 \text {. }
$$

Proof. From Lemma 1, we know that $x_{k} \in \mathscr{L}$ for all $k$. Since $f(x)$ is bounded below on $\mathscr{L}$, Lemma 1 means

$$
\lambda \sum_{r=0}^{k} \sigma\left(t_{r}\right) \leq\left(1+\mu_{0}\right)^{k+1} f\left(x_{0}\right)-f\left(x_{k+1}\right) .
$$

Then as $k \longrightarrow \infty$, we have

$$
\lambda \sum_{r=0}^{\infty} \sigma\left(t_{r}\right)<\infty
$$

so that

$$
\lim _{k \rightarrow \infty} \sigma\left(t_{k}\right)=0
$$

From Definition 1, we have

$$
\lim _{k \rightarrow \infty} t_{k}=\lim _{k \rightarrow \infty} \frac{-g_{k}^{T} d_{k}}{\left\|d_{k}\right\|}=0 .
$$

Using Assumption 2, we conclude

$$
\lim _{k \rightarrow \infty} \sigma\left(\left\|g_{k}\right\|\right)=0 \text {, }
$$

which implies $\lim _{k \longrightarrow \infty}\left\|g_{k}\right\|=0$. Thus, by Assumption 3, the proof is completed.

\section{Numerical Experiments}

In order to assess the computational efficiency of the presented method, we focus on providing some numerical results of our algorithm, denoted by ANLS, and compare the results with DFU-PRP in [4]. We tested algorithms by following a set of examples. Our algorithms stop if the termination condition $\left\|f_{k}\right\| \leq 10^{-11}$ is satisfied. All of the test problems are taken from [12] and the calculations were carried out using MATLAB.
Problem a. Problem 8

$$
\begin{aligned}
x_{1}^{2}+x_{2}^{2}-25 & =0, \\
x_{1} x_{2}-9 & =0, \\
x_{0} & =\left[1, \frac{1}{2}\right]^{T} .
\end{aligned}
$$

Problem $b$. Problem 26

$$
\begin{aligned}
\left(1+x_{2}^{2}\right)^{2} x_{1}+x_{3}^{4}-3 & =0, \\
x_{0} & =[-10,-10,-10]^{T} .
\end{aligned}
$$

Problem c. Problem 39

$$
\begin{aligned}
x_{2}-x_{1}^{3}-x_{3}^{2} & =0, \\
x_{1}^{2}-x_{2}-x_{4}^{2} & =0, \\
x_{0} & =[2,2,2,2]^{T} .
\end{aligned}
$$

Problem $d$. Problem 40

$$
x_{1}^{3}+x_{2}^{2}-1=0
$$

$$
\begin{aligned}
x_{1}^{2} x_{4}-x_{3} & =0, \\
x_{4}^{2}-x_{2} & =0, \\
x_{0} & =\left[1, \frac{1}{2}, \frac{1}{3}, \frac{1}{4}\right]^{T} .
\end{aligned}
$$

Problem e. Problem 61

$$
\begin{aligned}
3 x_{1}-2 x_{2}^{2}-7 & =0, \\
4 x_{1}-x_{3}^{2}-11 & =0, \\
x_{0} & =[0.1,0.1,0.1]^{T} .
\end{aligned}
$$

Problem f. Problem 81

$$
\begin{aligned}
x_{1}^{2}+x_{2}^{2}+x_{3}^{2}+x_{4}^{2}+x_{5}^{2}-10 & =0, \\
x_{2} x_{3}-5 x_{4} x_{5} & =0, \\
x_{1}^{3}+x_{2}^{3}+1 & =0, \\
x_{0} & =[-1,-1,-1,-1,-1]^{T} .
\end{aligned}
$$

Problem g. Problem 111 
TABLE 1: Numerical results.

\begin{tabular}{lcccc}
\hline Problems & $\begin{array}{c}n_{i} / n_{f} \\
(\mathrm{DFU}-\mathrm{PRP})\end{array}$ & $f\left(x^{*}\right)$ & $n_{i} / n_{f}(\mathrm{ANLS})$ & $f\left(x^{*}\right)$ \\
\hline$A$ & $14 / 17$ & $7.63 e-10$ & $7 / 10$ & $3.4862 e-07$ \\
$B$ & $78 / 210$ & $7.77 e-13$ & $13 / 14$ & $3.8652 e-07$ \\
$C$ & $250 / 717$ & $5.42 e-09$ & $5 / 6$ & $4.2731 e-06$ \\
$D$ & $\mathrm{~F} / \mathrm{F}$ & - & $3 / 4$ & $1.0868 e-06$ \\
$E$ & $\mathrm{~F} / \mathrm{F}$ & - & $4 / 6$ & $5.8224 e-07$ \\
$f$ & $\mathrm{~F} / \mathrm{F}$ & - & $8 / 12$ & $1.5056 e-07$ \\
$g$ & $358 / 1910$ & $4.99 e-09$ & $81 / 546$ & $4.8625 e-06$ \\
\hline
\end{tabular}

$$
\begin{aligned}
e^{x_{1}}+2 e^{x_{2}}+2 e^{x_{3}}+e^{x_{6}}+e^{x_{10}}-2 & =0, \\
e^{x_{4}}+2 e^{x_{5}}+e^{x_{6}}+e^{x_{7}}-1 & =0, \\
e^{x_{3}}+e^{x_{7}}+e^{x_{8}}+2 e^{x_{9}}+e^{x_{10}}-1 & =0, \\
x_{0} & =\left[1, \frac{1}{2}, \frac{1}{3}, \ldots, \frac{1}{10}\right]^{T} .
\end{aligned}
$$

Algorithm 1 takes advantages parameters $\sigma=10^{-5}$, $\rho=10^{-3}, \beta=0.6, \varepsilon=10^{-5}, c=0.9, \mu_{k}=\left\|g_{k}\right\| / k !, \lambda_{k r}=1 / \ell_{k}$, $J=7$, and $r=0,1, \ldots, \ell_{k}-1$. Let $\left(n_{f}\right)$ be the number of function evaluations and $\left(n_{i}\right)$ be the number of iterations as two cost measures to compare the proposed algorithms. Moreover, the sign $(F)$ means that the total number of the number of function evaluations and gradient evaluations exceeds 10,000 .

In this table, the values of the DFU-PRP method are according to the numerical result in the appendix table in [4]. It is obvious from Table 1 that the ANLS algorithm shows better performance than the DFU-PRP algorithm.

\section{Conclusions}

In this article, we propose a combined algorithm of the gradient and Newton's methods for solving a system of nonlinear equations. We have considered a new combination and different from others in the literature. The numerical results demonstrate that the developed algorithm is more effective than the other similar ones. We will extend this algorithm to the trust region method in our future research.

\section{Data Availability}

No data were used to support this study.

\section{Conflicts of Interest}

The authors declare that they have no conflicts of interest.
[2] Y. Mo, H. Liu, and Q. Wang, "Conjugate direction particle swarm optimization solving systems of nonlinear equations," Computers and Mathematics with Applications, vol. 57, no. 11-12, pp. 1877-1882, 2009.

[3] S. Taheri and M. Mammadov, "Solving systems of nonlinear equations using a globally convergent optimization algorithm," Global Journal of Technology and Optimization, vol. 3, pp. 132-138, 2012.

[4] D. Ataee Tarzanagh, P. Nazari, and M. Reza Peyghami, "A nonmonotone PRP conjugate gradient method for solving square and under-determined systems of equations," Computers \& Mathematics with Applications, vol. 73, no. 2, pp. 339-354, 2017.

[5] Dennis and R. B. Schnabel, Numerical Methods for Unconstrained Optimization and Nonlinear Equations, PrenticeHall, Englewood Cliffs, NJ, USA, 1996.

[6] R. Fletcher, Practical Methods of Optimization, John Wiley \& Sons, Hoboken, NJ, USA, 1987.

[7] Y. Yuan and W. Sun, Optimization Theory and Methods, Science Press, Beijing, China, 1997.

[8] L. Grippo, F. Lampariello, and S. Lucidi, "A truncated Newton method with nonmonotone line search for unconstrained optimization," Journal of Optimization Theory and Applications, vol. 60, no. 3, pp. 401-419, 1989.

[9] W. Sun, J. Han, and J. Sun, "Global convergence of nonmonotone descent methods for unconstrained optimization problems," Journal of Computational and Applied Mathematics, vol. 146, no. 1, pp. 89-98, 2002.

[10] M. Reza Peyghami and D. Ataee Tarzanagh, "A relaxed nonmonotone adaptive trust region method for solving unconstrained optimization problems," Computational Optimization and Applications, vol. 61, no. 2, pp. 321-341, 2015.

[11] $\mathrm{Z}$. Yu and D. Pu, "A new nonmonotone line search technique for unconstrained optimization," Journal of Computational and Applied Mathematics, vol. 219, no. 1, pp. 134-144, 2008.

[12] W. Hock and K. Schittkowski, "Test examples for nonlinear programming codes," Journal of Optimization Theory and Applications, vol. 30, no. 1, pp. 127-129, 1980.

\section{References}

[1] M. Abdollahi, A. Bouyer, and D. Abdollahi, "Improved cuckoo optimization algorithm for solving systems of nonlinear equations," The Journal of Supercomputing, vol. 72, no. 3, pp. 1246-1269, 2016. 